\title{
Turbo Equalization with Cancelation of Nonlinear Distortion Effects for CP-Assisted and Zero-Padded MC-CDMA Signals
}

\author{
R. Dinis, P. Silva and T. Araújo \\ IST, Tech. Univ. of Lisbon, Portugal
}

Abstract - We consider MC-CDMA schemes, with reduced envelope fluctuations. Both CP-assisted (Cyclic Prefix) and ZP (Zero-Padded) MC-CDMA schemes are addressed. We develop Turbo FDE (Frequency-Domain Equalization) schemes, combined with cancelation of nonlinear distortion effects. The proposed turbo receivers allow significant performance improvements at low and moderate SNR, even when the transmitted signals have reduced envelope fluctuations. ${ }^{1}$

\section{Introduction}

MC-CDMA schemes (Multicarrier Coded Division Multiple Access) combine the advantages of CDMA schemes with the advantages of OFDM modulations (Orthogonal Frequency Division Multiplexing). However, since the transmission over time-dispersive channels destroys the orthogonality between spreading codes, an FDE (Frequency-Domain Equalizer) is required before the de-spreading operation [1]. A promising iterative receiver for multicode MC-CDMA signals was proposed in [2], which have asymptotic performances that can be close to the single-code performance, provided that we have severely time-dispersive channels and the spreading factor is high. However, for moderate spreading factors the performances are rather poor, and far from the single-code performance. Moreover, the performance improvements as we increase the number of iterations are much lower for low and moderate SNR (Signal-to-Noise Ratio). An alternative to conventional CP-assisted MC-CDMA is ZP (Zero-Padded) MC-CDMA, where the CP samples are replaced by zeros [3].

As with other multicarrier schemes, MC-CDMA signals have strong envelope fluctuations and high PMEPR values (Peak-to-Mean Envelope Power Ratio) which makes them very prone to nonlinear effects. A promising approach to reduce the envelope fluctuations of the transmitted signals while maintaining the spectral occupation of conventional schemes is to employ clipping techniques, combined with a frequencydomain filtering [4], [5]. However, the nonlinear distortion effects can be severe when a low-PMEPR transmission is intended [4], [5]. To improve the performances, we can employ the receivers proposed in [6], [7] where the nonlinear distortion effects are iteratively estimated and compensated.

${ }^{1}$ This work was partially supported by the FCT project POSI/CPS/46701/2002 - MC-CDMA, the FCT/POCI 2010 research grant SFRH/BD/24520/2005 and the project C-MOBILE IST-2005-27423.
In this paper, we consider MC-CDMA schemes where all spreading codes are intended to a given user ${ }^{2}$. Both $\mathrm{CP}$-assisted MC-CDMA and ZP MC-CDMA schemes are considered, with IB-DFE receivers (Iterative Block Decision Feedback Equalization) based on the ones proposed in [2]. To reduce the envelope fluctuations of the transmitted signals while maintaining the spectral occupation of conventional MC-CDMA signals we employ the clipping and frequency-domain filtering techniques considered in [5]. To improve the performances at low and moderate SNRs we consider the use of turbo equalization schemes, where the equalization and channel decoding operations are repeated iteratively, sharing information between them [8]. We develop frequency-domain turbo equalizers for both $\mathrm{CP}$-assisted and ZP MC-CDMA schemes which, in the case of low-PMEPR MC-CDMA schemes, combine an iterative estimation and cancelation of nonlinear distortion effects. The proposed turbo receivers have relatively low complexity, since they allow FFT-based, frequency-domain implementations.

\section{CP-assisted MC-CDMA and ZP MC-CDMA}

\section{A. Conventional Transmitted Signals}

In this paper we consider MC-CDMA systems employing frequency-domain spreading. The frequency-domain block to be transmitted by the $m$ th MC-CDMA block is $\left\{S_{k}^{(m)} ; k=\right.$ $0,1, \ldots, N-1\}$, where $N=K M$, with $K$ denoting the spreading factor and $M$ the number of data symbols for each spreading code. The frequency-domain symbols are given by $S_{k}^{(m)}=\sum_{p=1}^{P} \xi_{p} S_{k, p}^{(m)}$, where $\xi_{p}$ is an appropriate weighting coefficient that accounts for the different powers assigned to different spreading codes (the power associated to the $p$ th spreading code is proportional to $\left|\xi_{p}\right|^{2}$ ) and $S_{k, p}^{(m)}=$ $C_{k, p}^{(m)} A_{k M o d M, p}^{(m)}$ is the $k$ th chip for the $p$ th spreading code ${ }^{3}$, where $\left\{A_{k, p}^{(m)} ; k=0,1, \ldots, M-1\right\}$ is the block of data symbols associated to the $p$ th spreading code and $\left\{C_{k, p}^{(m)} ; k=\right.$ $0,1, \ldots, N-1\}$ is the corresponding spreading sequence. An orthogonal spreading is assumed throughout this paper, with $C_{k, p}^{(m)}$ belonging to a QPSK constellation (Quaternary Phase

\footnotetext{
${ }^{2}$ These schemes are also denoted MC-CDM (MultiCarrier Code Division Multiplexing), since the data symbols are multiplexed by the different spreading codes and subcarriers.

${ }^{3}$ This means that the different chips associated to a different data symbol are uniformly spread within the transmission band.
} 
Shift Keying). Without loss of generality, it is assumed that $\left|C_{k, p}^{(m)}\right|=1$.

For CP-assisted MC-CDMA schemes, the transmitted signal associated to the $m$ th data block is given by

$$
s^{(m)}(t)=\sum_{n=-N_{G}}^{N-1} s_{n}^{(m)} h_{T}\left(t-n T_{S}\right),
$$

with $T_{S}$ denoting the sampling interval, $N_{G}$ denoting the number of samples at the cyclic prefix and $h_{T}(t)$ is the impulse response of the reconstruction filter. It is assumed that the timedomain block is periodic, with period $N$, i.e., $s_{-n}^{(m)}=s_{N-n}^{(m)}$. The first $N_{G}$ samples can be regarded as a CP for the MCCDMA block.

If we discard the samples associated to the $\mathrm{CP}$ at the receiver then there is no interference between blocks, provided that the length of the $\mathrm{CP}$ is higher than the length of the overall channel impulse response. Moreover, the linear convolution associated to the channel can be regarded as a cyclic convolution relatively to the $N$-length, useful part of the received block, $\left\{y_{n} ; n=0,1, \ldots, N-1\right\}$ (for the sake of notation simplicity, we will ignore the dependence with the block index $m$ in the following). This means that the corresponding frequency-domain block (i.e., the length- $N$ DFT (Discrete Fourier Transform) of the block $\left\{y_{n} ; n=0,1, \ldots, N-1\right\}$ ) is $\left\{Y_{k} ; k=0,1, \ldots, N-1\right\}$, where

$$
Y_{k}=S_{k} H_{k}+N_{k}
$$

with $H_{k}$ denoting the channel frequency response for the $k$ th subcarrier and $N_{k}$ the corresponding channel noise. Clearly, the impact of a time-dispersive channel reduces to a scaling factor for each subcarrier. However, there is a degradation $\eta_{G}=$ $N /\left(N+N_{G}\right)$, due to the "useless" power spent on the CP. Whenever the duration of the $\mathrm{CP}$ is a significant fraction of the block duration this degradation is not negligible. To avoid this degradation we will replace the CP by $N_{G}$ zero-valued samples, i.e., we will consider ZP MC-CDMA schemes.

Unfortunately, the linear convolution associated to the channel is no longer equivalent to a cyclic convolution and (2) does not hold for ZP MC-CDMA. Therefore, the low-complexity receiver adopted for conventional, CP-assisted MC-CDMA cannot be employed. By using overlap-and-add techniques, the receiver complexity is similar to the one of conventional $\mathrm{CP}$ assisted MC-CDMA schemes, but the performances are also identical [3].

\section{B. MC-CDMA Schemes with Reduced Envelope Fluctuations}

To reduce the envelope fluctuations of the transmitted signals, we can employ the transmitter structure depicted in fig. 1 , where a conventional CP-assisted MC-CDMA scheme is assumed (the extension to ZP MC-CDMA is straightforward). The transmitter structure of fig. 1 is based on the nonlinear signal processing schemes proposed in [4] for reducing the PMEPR of OFDM signals while maintaining the spectral efficiency of conventional OFDM schemes. Within that transmitter, $N^{\prime}-N$ zeros are added to the original frequencydomain block (i.e., $N^{\prime}-N$ idle subcarriers), followed by an IDFT operation so as to generate a sampled version of the time-domain MC-CDMA signal, with an oversampling factor $M_{T x}=N^{\prime} / N$. Each time-domain sample is submitted to a nonlinear device corresponding to an ideal envelope clipping, so as to reduce the envelope fluctuations on the transmitted signal. The clipped signal is then submitted to a frequencydomain filtering procedure, through the set of multiplying coefficients $\left\{G_{k} ; k=0,1, \ldots, N^{\prime}-1\right\}$, in order to reduce the out-of-band radiation levels inherent to the nonlinear operation.

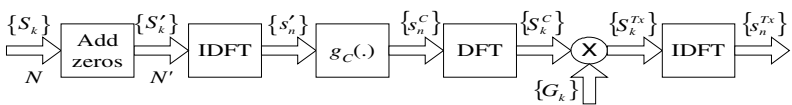

Fig. 1. Transmitter model for generating low-PMEPR MC-CDMA signals.

It is shown in [5] that the frequency-domain block to be transmitted $\left\{S_{k}^{T x}=S_{k}^{C} G_{k} ; k=0,1, \ldots, N^{\prime}-1\right\}$ can be decomposed into useful and nonlinear self-interference components:

$$
S_{k}^{T x}=\alpha S_{k}^{\prime} G_{k}+D_{k} G_{k},
$$

with $\alpha$ defined in [4], [5]. Throughout this paper we assume that $G_{k}=1$ for the $N$ in-band subcarriers and 0 for the $N^{\prime}-N$ out-of-band subcarriers. In this case, $S_{k}^{T x}=\alpha S_{k}^{\prime}+$ $D_{k}$ for the in-band region and 0 , otherwise. It can also be shown that $D_{k}$ is approximately Gaussian-distributed, with zero mean; moreover, $E\left[D_{k} D_{k^{\prime}}^{*}\right]$ can be computed analytically, as described in [4], [5].

\section{Conventional Receivers Structures}

Let us consider a CP-assisted MC-CDMA scheme. From (2), it is clear that the orthogonality between users is lost in frequency selective channels. For this reason, an FDE is required before the despreading operation [1]. This means that the receiver structure can be the one depicted in fig. 2.A. Under perfect channel estimation, the FDE coefficients are given by $F_{k}=H_{k}^{*} /\left(\beta+\left|H_{k}\right|^{2}\right)$, with $\beta=E\left[\left|N_{k}\right|^{2}\right] / E\left[\left|S_{k}\right|^{2}\right]$, which corresponds to the minimization of the MMSE in the frequency-domain samples $S_{k}$. The data symbols associated to the $p$ th spreading code can be estimated by despreading the samples at the FDE output $\tilde{S}_{k}=Y_{k} F_{k}$, i.e., from

$$
\tilde{A}_{k, p}=\sum_{k^{\prime} \in \Psi_{k}} \tilde{S}_{k^{\prime}} C_{k^{\prime}, p}^{*},
$$

with $\Psi_{k}$ denoting the set of frequencies employed to transmit the $k$ th data symbol of each spreading code (for a $K \times M$ interleaving, the set $\Psi_{k}$ is given by $\Psi_{k}=\{k, k+M, \ldots, k+$ $(K-1) M\})$.

Let us consider now ZP MC-CDMA schemes. Fig. 2.B shows the receiver structure that we are considering for $\mathrm{ZP}$ MC-CDMA schemes. Within this receiver, an augmented block $\left\{y_{n}^{(J)} ; n=0,1, \ldots, J N-1\right\}$ is generated by adding $J N-N_{G}-N$ zeros to the received time-domain block $\left\{y_{n}^{(J)} ; n=0,1, \ldots, N+N_{G}-1\right\}$ that includes the $N_{G}$ samples 


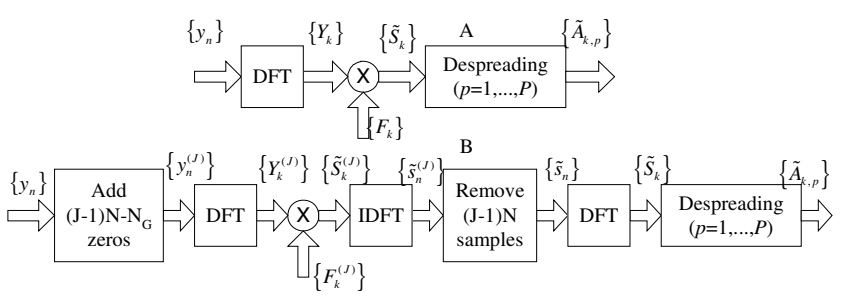

Fig. 2. Frequency-domain receiver for CP-assisted MC-CDMA (A) and ZP MC-CDMA (B).

associated to the "zero-padded region" immediately after the "useful" MC-CDMA block.

The DFT of the augmented time-domain block is computed, leading to the frequency-domain block $\left\{Y_{k}^{(J)} ; k=\right.$ $0,1, \ldots, J N-1\}$. If the duration of the "zero-padded region" is longer than the duration of the overall channel impulse response, the $N_{G}$ zeros before the "MC-CDMA block" can be regarded as a $\mathrm{CP}$ for the "augmented ZP MC-CDMA block" that includes the subsequent $N_{G}$ zeros. Therefore, a relation similar to (2) could be derived for the augmented frequencydomain block:

$$
Y_{k}^{(J)}=S_{k}^{(J)} H_{k}^{(J)}+N_{k}^{(J)},
$$

with $H_{k}^{(J)}$ and $N_{k}^{(J)}$ replacing $H_{k}$ and $N_{k}$ of (2), respectively. The equivalent transmitted frequency-domain block $\left\{S_{k}^{(J)} ; k=\right.$ $0,1, \ldots, J N-1\}$ is the DFT of the block $\left\{s_{n} r_{n} ; n=\right.$ $0,1, \ldots, J N-1\}$, where $r_{n}=1$ for $0 \leq n \leq N-1$ and 0 otherwise (once again, it is assumed that the samples $s_{n}$ are periodic with period $N$ ). Since the DFT of the block $\left\{s_{n}^{(J)} ; n=0,1, \ldots, J N-1\right\}$ (the block $\left\{s_{n} ; n=\right.$ $0,1, \ldots, N-1\}$ repeated $J$ times) is the block $\left\{S_{k}^{(J)} ; k=\right.$ $0,1, \ldots, J N-1\}$, with $S_{k}^{(J)}=J S_{k / J}$ for $k / J$ integer and 0 otherwise, then $S_{k}^{(J)}=\sum_{k^{\prime \prime}=0}^{N-1} J S_{k^{\prime \prime}} R_{k-J k^{\prime \prime}}$, with $\left\{R_{k} ; k=0,1, \ldots, J N-1\right\}=$ DFT $\left\{r_{n} ; n=0,1, \ldots, J N-\right.$ $1\}$. This means that there is intercarrier interference, which might lead to significant performance degradation. To cope with this intercarrier interference, the augmented block is submitted to a linear FDE, leading to the frequency-domain block $\left\{Y_{k, I g}^{(J)}=Y_{k}^{(J)} F_{k}^{(J)} ; k=0,1, \ldots, J N-1\right\}$, where $F_{k}^{(J)}$ denotes the $k$ th FDE coefficient. As with CP-assisted MC-CDMA these coefficients are optimized under the MMSE criterion, i.e., $F_{k}^{(J)}=H_{k}^{(J) *} /\left(\beta^{(J)}+\left|H_{k}^{(J)}\right|^{2}\right)$, with $\beta^{(J)}=$ $E\left[\left|N_{k}^{(J)}\right|^{2}\right] / E\left[\left|S_{k}^{(J)}\right|^{2}\right]$.

The signal component associated to the corresponding timedomain block, $\left\{\tilde{s}_{n}^{(J)} ; n=0,1, \ldots, J N-1\right\}$, is almost restricted to the first $N$ samples (it would be exactly restricted to these $N$ samples if the FDE coefficients were optimized under the $\mathrm{ZF}$ criterion (Zero Forcing)), and the remaining $(J-1) N$ samples are composed almost entirely by noise. For this reason, the last $(J-1) N$ samples are removed, leading to the final time-domain block at the FDE output $\left\{\tilde{s}_{n}=\tilde{s}_{n}^{(J)} ; n=\right.$ $0,1, \ldots, N-1\}$. The detection is based on the corresponding frequency-domain block $\left\{\tilde{S}_{k} ; k=0,1, \ldots, N-1\right\}=$ DFT $\left\{\tilde{s}_{n} ; n=0,1, \ldots, N-1\right\}$.

\section{IB-DFE Receivers}

\section{A. Linear Transmitters}

In this section we consider IB-DFE receivers based on the receiver proposed in [2]. Let us first consider $\mathrm{CP}$-assisted $\mathrm{MC}$ CDMA. For a given iteration, the output samples are given by [2]

$$
\tilde{S}_{k}=F_{k} Y_{k}-B_{k} \rho \hat{S}_{k}
$$

where $\left\{F_{k} ; k=0,1, \ldots, N-1\right\}$ and $\left\{B_{k} ; k=0,1, \ldots, N-\right.$ $1\}$ denote the feedforward and the feedback coefficients, respectively, and the correlation coefficient $\rho$, which can be regarded as the blockwise reliability of the decisions used in the feedback loop (from the previous iteration), is given by

$$
\rho=\frac{E\left[\hat{S}_{k} S_{k}^{*}\right]}{E\left[\left|S_{k}\right|^{2}\right]}=\sum_{p=1}^{P} \xi_{p}^{2} \frac{E\left[\hat{A}_{k, p} A_{k, p}^{*}\right]}{E\left[\left|A_{k, p}\right|^{2}\right]}=\sum_{p=1}^{P} \xi_{p}^{2} \rho_{p},
$$

with $\rho_{p}=E\left[\hat{A}_{k, p} A_{k, p}^{*}\right] / E\left[\left|A_{k, p}\right|^{2}\right] .\left\{\hat{S}_{k}=\sum_{p=1}^{P} \xi_{p} \hat{S}_{k, p} ; k=\right.$ $0,1, \ldots, N-1\}$, with $\hat{S}_{k, p}=\hat{A}_{k \operatorname{Mod} M, p} C_{k, p}$, where $\hat{A}_{k, p}$ denotes the estimate of $A_{k, p}$ from the previous iteration.

The optimum coefficients $F_{k}$ and $B_{k}$ that maximize the overall SNR in the samples $\tilde{S}_{k}$ are given by [2] ${ }^{4}$

$$
F_{k}=\frac{\kappa H_{k}^{*}}{\beta+\left(1-\rho^{2}\right)\left|H_{k}\right|^{2}},
$$

and $B_{k}=F_{k} H_{k}-1$, respectively, where $\beta=$ $E\left[\left|N_{k}\right|^{2}\right] / E\left[\left|S_{k}\right|^{2}\right]$ and $\kappa$ is selected so as to ensure that $\sum_{k=0}^{N-1} F_{k} H_{k} / N=1$.

For the first iteration, we do not have any information about $S_{k}$ and the correlation coefficient is zero. This means that the receiver reduces to a linear FDE optimized under the MMSE criterion [1]. After the first iteration, and if the residual BER is not too high (at least for the spreading codes for which a higher transmit power is associated), we have $\hat{A}_{k, p}=A_{k, p}$ for most of the data symbols, leading to $\hat{S}_{k} \approx S_{k}$. Consequently, we can use the feedback coefficients to eliminate a significant part of the residual interference.

The IB-DFE receiver can easily be extended to $\mathrm{ZP} \mathrm{MC}$ CDMA. The main difference is that the feedforward and feedback filtering operations use blocks with length $J N$ instead of the length- $N$ of CP-assisted MC-CDMA. Therefore, (6) takes the form

$$
\widetilde{S}_{k}^{(J)}=F_{k}^{(J)} Y_{k}-B_{k}^{(J)} \rho^{(J)} \hat{S}_{k}^{(J)}
$$

where $\rho^{(J)}=E\left[\hat{S}_{k}^{(J)} S_{k}^{(J) *}\right] / E\left[\left|S_{k}^{(J)}\right|^{2}\right]$,

$$
F_{k}^{(J)}=\frac{\kappa^{(J)} H_{k}^{(J) *}}{\beta^{(J)}+\left(1-\left(\rho^{(J)}\right)^{2}\right)\left|H_{k}^{(J)}\right|^{2}},
$$

with $\kappa^{(J)}$ selected so as to ensure $\sum_{k=0}^{J N-1} F_{k}^{(J)} H_{k}^{(J)} /(J N)=$ 1 , and $B_{k}^{(J)}=F_{k}^{(J)} H_{k}^{(J)}-1$.

${ }^{4}$ It should be noted that, contrarily to [2], we are considering a normalized feedforward filter. 


\section{B. Low-PMEPR MC-CDMA Schemes}

It was shown in [6], [7] that we can improve significantly the performance of OFDM schemes submitted to nonlinear devices by employing a receiver with iterative cancelation of nonlinear distortion effects. This concept can be extended to both $\mathrm{CP}$-assisted and ZP MC-CDMA schemes. For CPassisted MC-CDMA this leads to the receiver structure of fig. 3 (the same concept could be employed with ZP MC-CDMA schemes). The basic idea behind this receiver is to use an estimate of the nonlinear self-distortion $\left\{\hat{D}_{k} ; k=0,1, \ldots, N-1\right\}$ provided by the preceding iteration to remove the nonlinear distortion effects in the received samples. Therefore, the received frequency-domain block $\left\{Y_{k} ; k=0,1, \ldots, N-1\right\}$ is replaced by the corrected block $\left\{Y_{k}^{\text {Corr }} ; k=0,1, \ldots, N-1\right\}$, where

$$
Y_{k}^{C o r r}=\frac{1}{\alpha}\left(Y_{k}-H_{k} \hat{D}_{k}\right) .
$$

The remaining of the receiver is similar, but with $\beta=$ $E\left[\left|N_{k}\right|^{2}\right] /\left(E\left[\left|\alpha S_{k}\right|^{2}\right]+E\left[\left|D_{k}\right|^{2}\right]\right)$. For the first iteration, the expectations in $\beta$ can easily be obtained by using the analytical approach of [4], [5]; for the remaining iterations they have to be obtained by simulation.

For a given iteration, $\left\{\hat{D}_{k} ; k=0,1, \ldots, N-1\right\}$ can be estimated form the blocks $\left\{\hat{A}_{k, p} ; k=0,1, \ldots, M-1\right\}(p=$ $1,2, \ldots, P)$ as follows: each block $\left\{\hat{A}_{k, p} ; k=0,1, \ldots, M-\right.$ $1\}$ is re-spread to generate the "average chip block" $\left\{\hat{S}_{k, p} ; k=\right.$ $0,1, \ldots, N-1\}$; the "average transmitted block" $\left\{\hat{S}_{k} ; k=\right.$ $0,1, \ldots, N-1\}$ is formed, with $\hat{S}_{k}=\sum_{p=1}^{P} \xi_{p} \hat{S}_{k, p} ;\left\{\hat{S}_{k} ; k=\right.$ $0,1, \ldots, N-1\}$ is submitted to a replica of the nonlinear signal processing scheme employed in the transmitter so as to form the "average transmitted block" $\left\{\hat{S}_{k}^{T x} ; k=0,1, \ldots, N-1\right\}$; $\hat{D}_{k}$ is given by $\hat{D}_{k}=\hat{S}_{k}^{T x}-\alpha \hat{S}_{k}$.

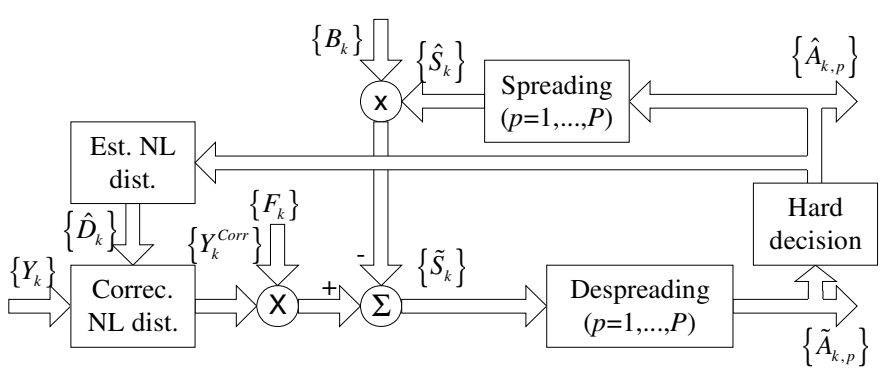

Fig. 3. IB-DFE receiver with cancelation of nonlinear distortion effects for CP-assisted MC-CDMA.

\section{Turbo Equalizer for MC-CDMA}

In this section we show how we can define Turbo FDE receivers based on the IB-DFE receivers. For the sake of simplicity, we will consider a CP-assisted MC-CDMA scheme (the generalization to ZP MC-CDMA schemes is straightforward).

(6) could be written as

$$
\tilde{S}_{k}=F_{k} Y_{k}-B_{k} \bar{S}_{k}
$$

with $\bar{S}_{k}=\rho \hat{S}_{k}$. Since $\rho$ can be regarded as the blockwise reliability of the estimates $\hat{S}_{k}, \bar{S}_{k}$ is the overall block average of the $S_{k}$ at the FDE output. To improve the performances, we could replace the "blockwise averages" by "symbol averages", which can be done as described in the following.

Let us assume that the transmitted symbols are selected from a QPSK constellation under a Gray mapping rule (the generalization to other cases is straightforward). We will define $A_{k, p}= \pm 1 \pm j=A_{k, p}^{I}+j A_{k, p}^{Q}$, with $A_{k, p}^{I}=\operatorname{Re}\left\{A_{k, p}\right\}$ and $A_{k, p}^{Q}=\operatorname{Im}\left\{A_{k, p}\right\}, k=0,1, \ldots, M-1 ; p=1,2, \ldots, P$, (similar definitions can be made for $\tilde{A}_{k, p}, \hat{A}_{k, p}$ and $\bar{A}_{k, p}$ ).

The LLRs (LogLikelihood Ratios) of the "in-phase bit" and the "quadrature bit", associated to $A_{k, p}^{I}$ and $A_{k, p}^{Q}$, respectively, are given by $L_{k, p}^{I}=2 \tilde{A}_{k, p}^{I} / \sigma_{p}^{2}$ and $L_{k, p}^{Q}=2 \tilde{A}_{k, p}^{Q} / \sigma_{p}^{2}$, respectively, where

$$
\sigma_{p}^{2}=\frac{1}{2} E\left[\left|A_{k, p}-\tilde{A}_{k, p}\right|^{2}\right] \approx \frac{1}{2 M} \sum_{k=0}^{M-1} E\left[\left|\hat{A}_{k, p}-\tilde{A}_{k, p}\right|^{2}\right] .
$$

It can be shown that the mean value of $A_{k, p}$ is

$$
\bar{A}_{k, p}=\tanh \left(\frac{L_{k, p}^{I}}{2}\right)+j \tanh \left(\frac{L_{k, p}^{Q}}{2}\right),
$$

and $\bar{S}_{k}$ can then be obtained form the $\bar{A}_{k, p}$.

The feedforward coefficients are still obtained from (8), with the blockwise reliability given by (7) and $\rho_{p}$ given by

$$
\rho_{p}=\frac{1}{2 M} \sum_{n=0}^{M-1}\left(\left|\operatorname{Re}\left\{\bar{A}_{k, p}\right\}+\right| \operatorname{Im}\left\{\bar{A}_{k, p}\right\} \mid\right) .
$$

The "overall chip averages" are then given by

$$
\bar{S}_{k}=\sum_{p=1}^{P} \xi_{p} C_{k, p} \bar{A}_{k \operatorname{Mod} M, p} .
$$

The soft decisions based on the "symbol averages", $\bar{A}_{k, p}$, can also be used in the estimation of the "average nonlinear distortion" components, $\bar{D}_{k}$, which are obtained as $\hat{D}_{k}$, but with $\bar{A}_{k, p}$ replacing $\hat{A}_{k, p}$.

Therefore, the receiver with "blockwise reliabilities", denoted in the following as IB-DFE with hard decisions, and the receiver with "symbol reliabilities", denoted in the following as IB-DFE with soft decisions, employ the same feedforward coefficients; however, in the first the feedback loop uses the "hard-decisions" on each data block, weighted by a common reliability factor, while in the second the reliability factor changes from symbol to symbol (in fact, the reliability factor is different in the real and imaginary component of each symbol).

We can also define a Turbo FDE that, as conventional the turbo equalizers, employs the channel decoder outputs instead of the uncoded "soft decisions" in the feedback loop. The receiver structure is similar to the IB-DFE with soft decisions, but with a SISO channel decoder (Soft-In, Soft-Out) employed in the feedback loop. The SISO block, that can be implemented as defined in [9], provides the LLRs of both the "information bits" and the "coded bits". The input of the SISO block are 
LLRs of the "coded bits" at the deinterleaver output ${ }^{5}$. Once again, the feedforward coefficients are obtained from (8) (or (10)), with the blockwise reliability given by (15).

\section{Performance Results}

In this section, we present a set of performance results concerning the proposed receiver structure. We consider both CP-assisted MC-CDMA and ZP MC-CDMA schemes with $N=256$ subcarriers and QPSK constellations. The BER performances are expressed as a function of $E_{b} / N_{0}$, with $N_{0}$ denoting the one-sided power spectral density of the channel noise and $E_{b}$ the "total $E_{b}$ ", including the energy spent on the CP. We consider a severely time-dispersive channel and the duration of the $\mathrm{CP}$ or $\mathrm{ZP}$ part of the block is identical to the duration of the useful part of the block. This means that, for the CP-assisted MC-CDMA scheme, the performance degradation due to the "useless" power spent on the CP is $3 \mathrm{~dB}$, since $\eta_{G}=1 / 2$ (similar results were obtained for other severely time-dispersive channels and other values of $\eta_{G}$ ). We use the $P=K$ available spreading codes, corresponding to a fully loaded scenario. We adopted a 64-state, rate-1/2 convolutional code with generators $1+D^{2}+D^{3}+D^{5}+D^{6}$ and $1+D+D^{2}+D^{3}+D^{6}$. We consider perfect synchronization and channel estimation conditions.

Let us first consider linear transmitters and uncoded BER performances. Figs. 4 and 5 concern IB-DFE receivers, with either hard decisions or soft decisions, when $K=16$ and $K=256$, respectively. From these figures, it is clear that $\mathrm{ZP}$ MC-CDMA outperforms CP-assisted MC-CDMA, although its advantage is mainly due to the fact that no useless power is spent on the CP. By using the iterative receiver we can improve significantly the performances of both modulation schemes. The use of soft decisions leads to a small improvement, that is only visible when $K=16$. As expected, the performances with $K=16$ are much worse than the performances with $K=256$.

Let us consider now the impact of channel coding, still with linear transmitters. For IB-DFE schemes, a soft-decision Viterbi decoder was employed; for turbo schemes, we adopted a SISO decoder implemented using the "Max-log-MAP approach" [9]. Fig. 6 concern the case when $K=16$ (contrarily to the uncoded case, the performance is similar with $K=16$ and $K=256$ ). Clearly, the use of turbo FDE receivers lead to significant performance improvements relatively to the IB-DFE receivers, which increase as we increase the number of iterations: about $2 \mathrm{~dB}$ after 3 iterations, for both CP-assisted MC-CDMA and ZP MC-CDMA. Once again, ZP MC-CDMA outperforms CP-assisted MC-CDMA, with its advantage mainly due to the fact that no useless power is spent on the CP.

Let us consider now low-PMEPR MC-CDMA schemes. The normalized clipping level is $s_{M} / \sigma=0.3$ (naturally, $s_{M} / \sigma=$ $+\infty$ corresponds to a linear transmitter) and $G_{k}=1$ for the

\footnotetext{
${ }^{5}$ As usual, it is assumed that the bits at the channel encoder output are interleaved before being mapped into the adopted constellation.
}

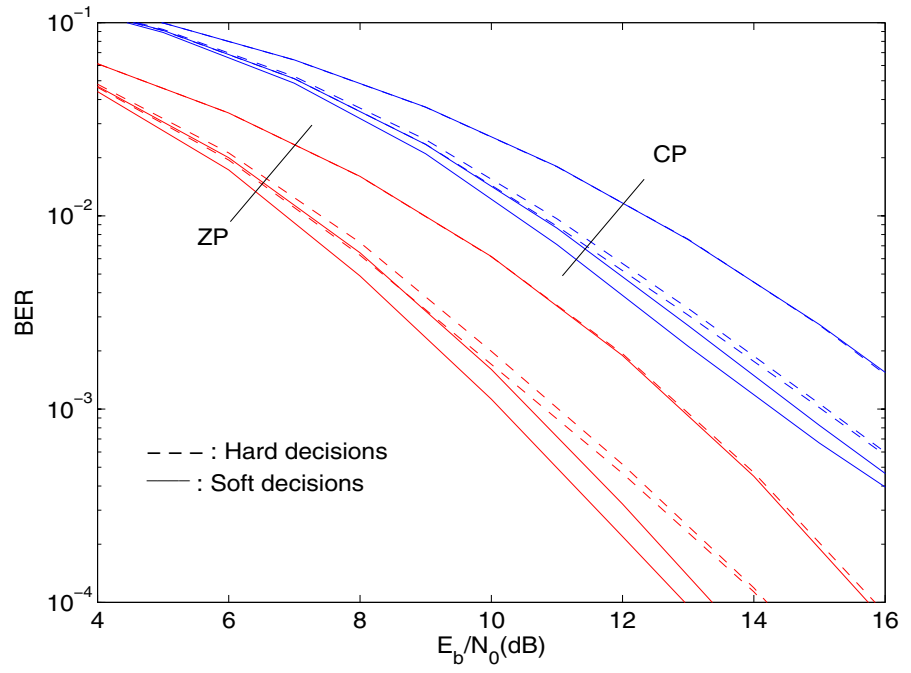

Fig. 4. Uncoded BER performances for IB-DFE receivers with 1,2 and 4 iterations (improved performances as we increase the iteration order), when $K=16$.

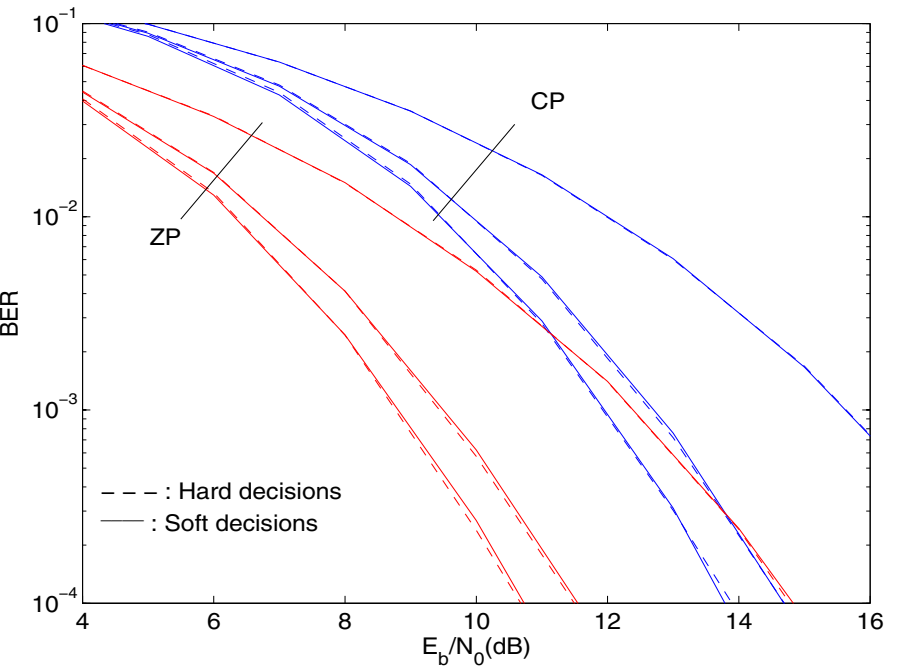

Fig. 5. As in fig. 4, but for when $K=256$.

$N$ in-band subcarriers and 0 for the remaining subcarriers. With this technique, and CP-assisted MC-CDMA, the PMEPR of the transmitted signals is $4.1 \mathrm{~dB}$ (for conventional MCCDMA signals the PMEPR is $8.4 \mathrm{~dB}$ [5]); for conventional ZP MC-CDMA and low-PMEPR ZP MC-CDMA schemes the PMEPR values are increased by a factor $10 \log _{10}=$ $\left(\left(N+N_{G}\right) / N\right)=-10 \log _{10}\left(\eta_{G}\right)(3 \mathrm{~dB}$, in our case), since no signal is transmitted in the $\mathrm{ZP}$ region.

In fig. 7 we present the uncoded BER performances (averaged over all spreading codes) of IB-DFE receivers with soft decisions, for $K=P=16$ (a similar behavior was observed for $K=P=256$, although with better performances). From this figure, it is clear that the iterative receivers are especially interesting in the presence of nonlinear distortion effects, although there is still a significant degradation relatively to the case with linear transmitters. Moreover, for low SNR the performance degrades as we increase the number of iterations.

Let us consider now the impact of channel coding. Since the performance of IB-DFE receivers is poor at low SNR (the 


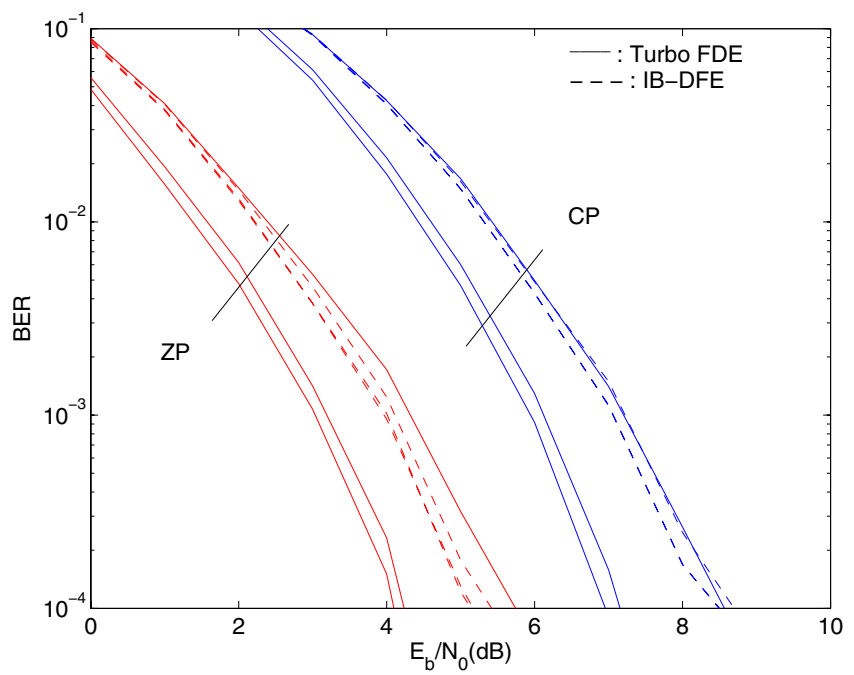

Fig. 6. Coded BER performances for turbo and IB-DFE receivers with 1,2 and 4 iterations (improved performances as we increase the iteration order), when $K=16$.

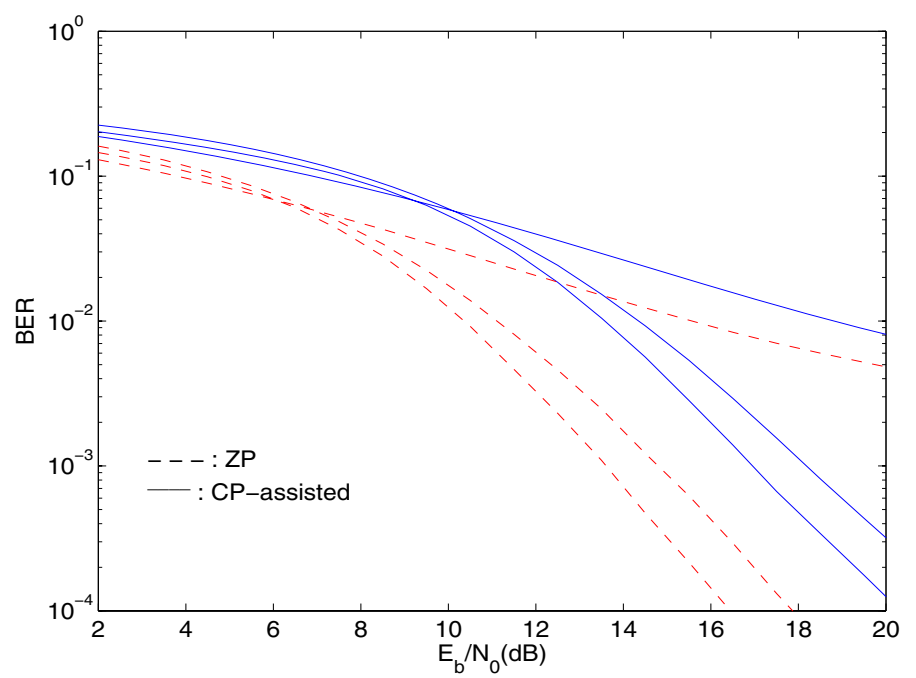

Fig. 7. Uncoded BER performances for IB-DFE receivers with iterative estimation and cancelation of nonlinear distortion effects and 1, 2 or 3 iterations (improved performances as we increase the iteration order).

typical working region when suitable channel coding schemes are employed), we will not consider the IB-DFE receivers, only the Turbo FDE receivers. Fig. 8 concerns the case when $K=$ $P=16$ (similar results were obtained with $K=P=256$ ). Clearly, the use of Turbo FDE receivers leads to significant performance improvements relatively to the IB-DFE receivers, which increase as we increase the number of iterations. The degradation due to nonlinear distortion effects is about 2 to 2.5 $\mathrm{dB}$ after 3 iterations.

It should be pointed out that performances could be improved if the convolutional code was replaced by a more powerful turbo code, provided that the data blocks were larger (naturally, this could mean splitting each coded data block by several FFT blocks, with the associated detection delay [10]). In this case, the degradation due to nonlinear distortion effects is even lower.

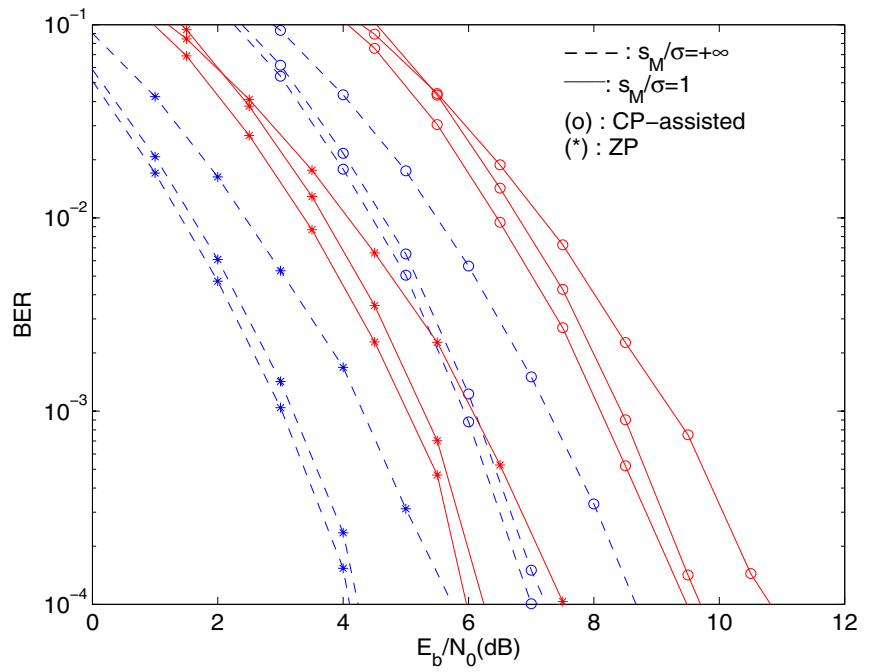

Fig. 8. Coded BER performances for Turbo FDE receivers with iterative estimation and cancelation of nonlinear distortion effects and 1, 2 or 3 iterations (improved performances as we increase the iteration order).

\section{Conclusions}

In this paper, we considered CP-assisted MC-CDMA and ZP MC-CDMA schemes and we developed FFT-based, frequencydomain turbo equalization schemes. We also considered lowPMEPR schemes where clipping techniques combined with frequency-domain filtering are employed at the transmitter. The proposed receivers allow significant performance improvements at low and moderate SNR, even when a low-PMEPR MC-CDMA transmission is intended. The receivers for ZP MC-CDMA are of special interest for systems where the duration of the channel impulse response is not a small fraction of the duration of the MC-CDMA block.

\section{References}

[1] H. Sari, "Orthogonal Multicarrier CDMA and its Detection on Frequency-Selective Channels", European Trans. on Telecomm., Vol. 13, No. 5, pp. 439-445, Sep.-Oct. 2002.

[2] R. Dinis, P. Silva and A. Gusmão, "Iterative Block Decision Feedback Equalization for Multicarrier CDMA", IEEE VTC'05(Spring), Stockholm, Sweden, May 2005.

[3] B.Muquet, M.Courville, G.Giannakis, Z.Wang and P.Dunamel, "Reduced Complexity Equalizers for Zero-Padded OFDM Transmission”, IEEE ICASSP 'O0, Vol. 5, pp. 2973-2976, June 2000.

[4] R. Dinis and A. Gusmão, "A Class of Nonlinear Signal Processing Schemes for Bandwidth-Efficient OFDM Transmission with Low Envelope Fluctuation", IEEE Trans. on Comm., Vol. 52, No. 11, pp. 2009-2018, Nov. 2004.

[5] R. Dinis and P. Silva, "Analytical Evaluation of Nonlinear Effects in MC-CDMA Signals", WOC'05, July 2005.

[6] J. Tellado, L. Hoo and J. Cioffi, "Maximum Likelihood Detection of Nonlinearly Distorted Multicarrier Symbols by Iterative Decoding”, IEEE Trans. on Comm. Vol. 51, Feb. 2003.

[7] A.Gusmão and R.Dinis, "Iterative Receiver Techniques for Cancellation of Deliberate Nonlinear Distortion in OFDM-type Transmission", Int. OFDM Workshop'04, Dresden, Sep. 2004.

[8] M. Tüchler, R. Koetter and A. Singer, "Turbo Equalization: Principles and New Results", IEEE Trans. on Comm., May 2002.

[9] B.Vucetic and J.Yuan, Turbo Codes: Principles and Applications, Kluwer Academic Publ., 2002.

[10] A.Gusmão and R.Dinis and P.Torres, "Low-PMEPR OFDM Transmission with an Iterative Receiver Technique for Cancellation of Nonlinear Distortion", IEEE VTC'05(Fall), Sep. 2005. 